\title{
CALIBRATION OF THE THERMAL INFRARED SENSOR ON THE LANDSAT DATA CONTINUITY MISSION
}

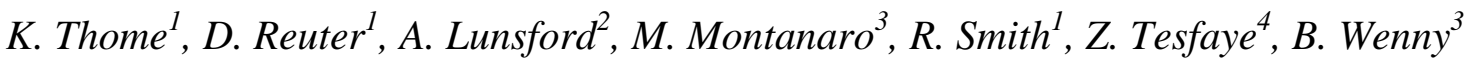 \\ ${ }^{1}$ NASA/GSFC, ${ }^{2}$ Catholic University, ${ }^{3}$ SigmaSpace, ${ }^{4}$ Bastion Technologies, Inc.
}

\begin{abstract}
The Landsat series of satellites provides the longest running continuous data set of moderate-spatial-resolution imagery beginning with the launch of Landsat 1 in 1972 and continuing with the1999 launch of Landsat 7 and current operation of Landsats 5 and 7[1]. The Landsat Data Continuity Mission (LDCM) will continue this program into a fourth decade providing data that are keys to understanding changes in land-use changes and resource management. LDCM consists of a two-sensor platform comprised of the Operational Land Imager (OLI) and Thermal Infrared Sensors (TIRS). A description of the applications and design of the TIRS instrument is given as well as the plans for calibration and characterization. Included are early results from preflight calibration and a description of the inflight validation.
\end{abstract}

Index Terms - Radiometric calibration, TIRS, LDCM

\section{INTRODUCTION}

The Landsat Data Continuity Mission (LDCM) will continue a fourth decade of Landsat data dating back to 1972 with the Landsat 1 through the current operations of Landsats 5 and 7 [1]. The goal of LDCM is to provide data to understand changes in land-use changes and resource management. LDCM consists of a two-sensor platform comprised of the Operational Land Imager (OLI) and Thermal Infrared Sensors (TIRS) with OLI operating in concert with, but independent from, TIRS. Both the OLI and TIRS sensors rely on a pushbroom approach with roughly 185-km swaths with OLI covering the reflective portion of the spectrum and TIRS the thermal emissive.

A large part of the success of the Landsat program is attributed to the emphasis placed on knowledge of the calibration of the sensors, both geometric and radiometric [2][3]. The radiometric assessment of the sensors has relied on a combination of prelaunch and post-launch efforts using laboratory, on-board, and vicarious calibration methods. LDCM continues the emphasis on calibration, with rigorous attention to NIST-traceability of the radiometric calibration, knowledge of out-of-band spectral response, and characterizing and minimizing stray light.
The current work describes the methods and facilities planned for the calibration of TIRS. The following section gives further information on the TIRS sensor followed by an overview of the calibration methodology. A description of the testing facilities at NASA GSFC being used for TIRS is given along with a preliminary error budget for the absolute radiometric calibration which shows that the required $2 \%$ radiometric accuracy for scene temperatures between 260 and $330 \mathrm{~K}$ is well within the capabilities of the system.

\section{TIRS DESCRIPTION}

The inclusion of TIRS on LDCM provides data to monitor water consumption on a field-by-field basis in the western United States. Other applications include examination of urban heat islands, mapping sensible heat flux, volcanic surveillance, and monitoring fire-induced vegetation depletion through burnt area mapping. To do this, TIRS will operate on the same platform as OLI but as an independent instrument. Data products from TIRS and OLI will be processed into a single data product at the United States Geological Survey Earth Resources Observation and Science facility. The radiometric and geometric calibration of TIRS provides the information necessary to combine TIRS and OLI data in a fashion that is seamless to the user.

TIRS is a two-band sensor (10.8 and 12 micrometer) with a $185-\mathrm{km}$ swath and $100-\mathrm{m}$ spatial resolution that is being built in-house at the NASA Goddard Space Flight Center. The focal plane array (FPA) for the pushbroom system is based on quantum well infrared photodetectors (QWIPs). Interference filters installed on the FPA provide spectral selection in such a way that guarantees data from the two bands are collected within 2.5 second of each other.

Three sub-chip assemblies (SCAs) QWIP arrays are needed to cover the entire swath of TIRS. Each SCA is a fully-populated 2-D array over which the spectral filters for each band are placed along with an aluminum mask to limit stray light. Two rows of detectors are selected from each band as well as two rows from under the mask to give a total of six rows of data compiled to give a TIRS image. The FPA is cryo-cooled to a stable temperature with an optical assembly that is passively cooled. A scene-select mirror rotates around the optical axis on a 45-degree plane to provide the telescope with a view to nadir (earth), space 
(cold calibration "target”), and on-board blackbody (hot calibration target).

A key component for the TIRS sensor is the onboard calibrator. The calibrator is a curved-plate blackbody. The surface of the plate is machined with $\mathrm{V}$-grooves to improve the overall emissivity of the plate. The design and coating are very similar to that used for MODIS to give high emissivity and controllable temperature. The output from the blackbody will be NIST traceable and capable of providing sources of varying temperature.

\section{CALIBRATION OVERVIEW}

TIRS continues Landsat's emphasis on calibration, with attention to NIST-traceability and instrument design necessary to meet the quality of Landsat heritage. The radiometric assessment of the sensor relies on a combination of prelaunch and post-launch efforts using laboratory, onboard, and vicarious calibration methods (where vicarious calibration refers to any method not relying on on-board calibrators). TIRS performance will be evaluated at the component, subsystem and system level. All acceptance testing and calibration will be done at GSFC including verification of vendor-level testing. Instrument-level requirements play a strong role in definition of the calibration test equipment used for the radiometric and spatial calibration in the thermal/vacuum chamber.

All radiometric acceptance testing and calibration will employ NIST-traceable standards. Figure 1 shows a block diagram overview of where National Institute of Standards and Technology) NIST standards enter the calibration path of TIRS. NIST standards are used to calibrate the laboratory blackbody source to a well-understood accuracy. The calibration of the laboratory blackbody is transferred to the TIRS sensor which in turn transfers the NIST standard to the on-board blackbody as described in Section 4. The onboard calibrator is the primary calibration of the TIRS sensor on orbit. As can be seen in the figure, the use of NIST standards and facilities occurs only in the calibration of the laboratory cavity blackbody.

Figure 1. Schematic of inclusion of NIST standards
The TIRS radiometric response is determined via the prelaunch characterization relative to the laboratory blackbody. This approach provides the highest accuracy calibration. The calibration philosophy is then to evaluate (or validate) the calibration parameters once TIRS is on orbit. If the calibration of TIRS is demonstrated to change significantly while on orbit using measurements during the checkout period, then the on-board blackbody (OBB) will be used as the primary pathway to NIST traceability.

A unique approach to preflight calibration is planned for TIRS in an effort to optimize the development schedule. A first round of calibration and characterization occurs shortly after the telescope optics are mated with the flight FPA, including spectral filters, and flight electronics. The advantage to Calibration 1 is the amount of testing time available permits the level of testing to cover the ranges of radiance levels and fields of view necessary for the understanding of the TIRS instrument. All measurements made are the full set of collections (fields of views, radiance levels, etc.). Testing of both electronics sides (A and B) as well as variations of the FPA and FPE temperatures occur in Calibration 1. The end result of the Calibration 1 testing is a TIRS instrument model that provides the capability to predict TIRS output for arbitrary instrument conditions and incident radiance fields.

A second round of testing occurs prior to vibration testing of the full-up TIRS instrument including the sceneselect mirror (SSM), main electronic box (MEB), cryocooler and harness, and OBB. Calibration 2 gives the first opportunity to characterize TIRS at the instrument level. All of the tests done in Calibration 1 are repeated in Calibration 2 but for a subset of conditions and FPA locations. In addition, a new set of tests for the OBB calibration are performed. The measurements are sufficient to evaluate the impact of the SSM, characterize the OBB, and transfer the calibration of the laboratory sources to the OBB. The emphasis in Calibration 2 is geometric characterizations to be evaluated post-vibration. Testing of both sides A and B takes place, but FPA/FPE temperatures are not varied.

Calibration 3 repeats all tests done during Calibration 2 to evaluate the impact of the vibration testing. The measurements collected must be sufficient to evaluate the impact of the vibration test on the spatial and geometric characteristics of the test unit, evaluate any temporal changes in the $\mathrm{OBB}$, and again transfer the calibration of the laboratory sources to the OBB. Testing of both sides A and $\mathrm{B}$ take place, but variations of FPA/FPE temperatures will not occur. Testing during Calibration 3 must be sufficient to allow verification of all Level 4 requirements.

\section{PRELAUNCH CALIBRATION}

Characterizing and calibrating TIRS couples results from Calibration 1 with component-level data to provide a wellunderstood instrument model that leads to instrument-level 
requirements verification. The instrument model receives rigorous evaluation during the pre- and post-vibration calibrations. In addition, the three separate calibration tests provide the opportunity to understand the temporal behavior of TIRS throughout the build process including tests that are suitable to be done at the platform level. Development of the testing plan for TIRS relied on a set of basic characterizations related to the radiometric, spatial, geometric, and spectral testing of TIRS.

A focus test is performed to ensure that the measured spot size across the TIRS focal plane matches the predicted size to within the measurement uncertainty. The test consists of translating a two-pixel, circular source along the optical axis of the CGSE (z-axis). Focus is obtained when the image size from the source is minimized. Z-axis translation is done for three locations for both bands for all three SCAs. The information from the initial focus test determines the best location for the FPA. Subsequent focus tests ensure that the TIRS instrument maintains its optical integrity during the build process.

Spatial shape testing evaluates the spatial resolution and must be done both pre-vibration and post-vibration. The measurement consists of scanning an edge source across and along the FPA while stopping at specified intervals to acquire imagery from TIRS. These data are converted to an equivalent line source image for an individual pixel. Multiple pixels across all three SCAs, for both bands will be evaluated. Data collected during the initial calibration work will make use of the 2-D aspect of the QWIP arrays to provide results based on both the translation of the knife edge as well from single images making use of multiple rows of detectors. Including measurements with a small-sized, two-pixel source prior to and after the scanning of the largesized aperture provides data sufficient to evaluate the geometric distortion of TIRS (or equivalently the imaged source size) changes across the field of view of TIRS.

Uniformity testing develops corrections for banding, streaking, and other similar detector-to-detector effects. Such effects are caused by the detectors, the instrument electronics, and to a lesser extent the optical elements. A full-aperture, full-field source, called the flood source, is used for uniformity evaluation. Collections are made at multiple temperatures with the source centered on the optical axis as well as at a variety of other locations to allow the non-uniformity of the source to be removed from the uniformity assessment. Periodic uniformity tests evaluate the health of the detector package (including electronics) from sub-assembly to instrument level.

Bright target recovery testing assesses the impact that a small-sized, high-temperature source has on the imaging by nearby pixels. The test determines electronic and detector effects that might impact the use of imagery near fires or similar high-temperature sources. Translation of a singlepixel, high-temperature source across the focal plane provides the necessary data to determine when the TIRS signal returns to background levels.

Spectral shape testing requires a monochromator assembly. Testing of the response of all detectors takes place at the component level while testing during the three phases of calibration test subsets of the detectors used by TIRS. Component-level tests provide the data needed to extrapolate the subset of results in the horizontal configuration to the full focal plane. Two levels of spectral testing are done: 1) in band and 2) out of band

Stray light, scattered light, and ghosting tests provide similar information with subtle differences related to the cause of the spurious light. Stray light tests consist of moving the flood source into the FOV of TIRS while measuring the radiance from a small-aperture source. The dominant cause of this effect is expected to be interactions between the incoming radiance field and optical and mechanical structures of TIRS. Scatter light and ghosting are examined by scanning a smaller, higher-radiance source across the FPA as well as regions beyond the detectors used for imaging. For example, edges of the aluminum mask covering the SCAs will be illuminated to determine areas of the FPA affected by scattered light.

The radiometric test evaluates the accuracy and precision of the conversion of sensor output to physical properties including effects from operating temperatures, electronics, and temporally-varying effects. In broad terms, the radiometric test is needed to derive the conversion equation from TIRS output to incident spectral radiance. Such conversion, at a minimum, requires knowledge of linearity, system offset, and system gain. Additional radiometric collections are used to evaluate noise, stability, banding, and streaking. Noise tests provide understanding of the NEdT model making use of flood source configuration and cavity blackbody source. Stability testing provides understanding of the sensor temporal behavior that allows accurate collection of the geometric and spatial data sets that require longer data-collection times. Stability issues (excluding those caused by the calibration sources) are caused by the detectors and the instrument electronics.

Of the tests listed above focus, uniformity, scatter/ghosting/straylight, and spatial shape/geometric distortion are done at the nominal FPA/FPE temperatures and for side A electronics only. A large fraction of the testing for spectral shape is done at nominal FPA/FPE temperatures but a small subset of testing is done for the inband spectral shape at varying FPA temperatures. Bright target recovery and radiometric testing cover a range of FPA/FPE temperatures and both sides A and B. Five FPA temperatures evaluate the FPA temperature's effect on the radiometric performance while three FPE temperatures are evaluated from a calibration standpoint.

As described above, characterization of the OBB takes place during Calibration 2 and relies on TIRS as a transfer radiometer to transfer the NIST-traceability of the laboratory 
blackbody to the OBB. The transfer calibration takes place during the radiometric calibration portion of Calibration 2 . Measurements for the OBB consist of illuminating portions of the FPA with the laboratory blackbody and then illuminating the FPA with the onboard blackbody. A 1degree by 1-degree source is used to permit the maximum number of detectors to be illuminated with the minimum number of pointings.

In order for the onboard blackbody calibrator (OBC) to be a viable calibration source it must also be calibrated in the prelaunch phase. The basic approach is shown schematically in Figure 2 and effectively uses the TIRS sensor as a transfer radiometer to transfer the calibration of the laboratory blackbody to the onboard.

Data from TIRS while viewing the laboratory blackbody gives a digital number (DN) output for that source (Lab. BB $\mathrm{DN})$. Combining this output with that from an appropriately cold source (Low temp. DN) gives the information needed to determine the radiometric calibration of TIRS. The sceneselect mirror is rotated to permit a view of the OBC leading to an OBC BB DN. Conversion of the DN to radiance gives an OBC-leaving radiance which, when coupled with the temperature readout of the OBB permits determination of an effective emissivity for the OBB.

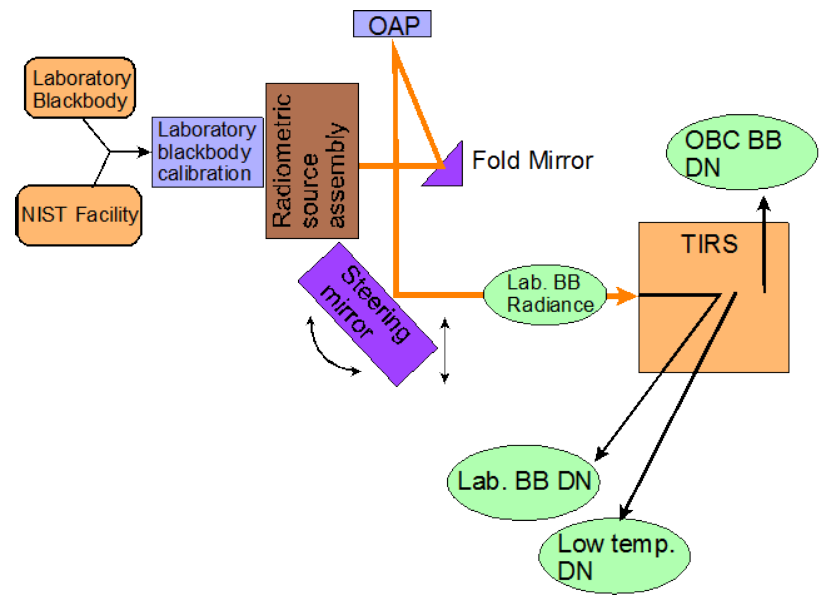

Figure 2. Diagram illustrating the calibration of the $\mathrm{OBC}$ using TIRS as a transfer standard.

\section{TIRS CALIBRATION SYSTEM}

The calibration testing of the TIRS takes place at Goddard Thermal/Vacuum Testing Facility and a calibration system has been acquired specifically for the testing of TIRS. The basis of the calibration system is a variable temperature, cavity blackbody source with variable aperture. A flood source, monochromator source, and cold background source are also included in the facility. Additional optical elements couple the monochromator to the collimating optics of the blackbody. Aperture, filter, and chopper wheels further condition the beam to provide the beam shape, magnitude, and frequency needed to test TIRS in a proper fashion. All pieces of the calibration equipment except the monochromator are placed inside the chamber on a single $\mathrm{LN}_{2}$ vibration isolated bench and enclosed within a cooled envelope to avoid scattering. The blackbody source has a documented calibration traceable to the NIST.

\section{IN-FLIGHT CALIBRATION}

The post-launch calibration of TIRS makes use of both onboard calibration information via the onboard calibrator as well as instrument telemetry and similar ancillary information. Other activities rely on vicarious methods using earth-scene data, lunar views, and deep-space looks.

The transfer of the radiometric calibration relies on demonstrating the validity of the onboard blackbody. The first step in the process is to evaluate the deep space view when on orbit. A shift in the expected value from preflight to on-orbit is indicative of a shift in the sensor behavior requiring an understanding of the sensor. In either case, the second step is to evaluate the sensor's output while viewing the OBB. A shift from the expected results while viewing the $\mathrm{OBB}$ would be indicative of either a change in the OBB or in the sensor. Reliance on vicarious calibration data will be needed to determine the root cause of the change (either sensor or $\mathrm{OBB}$ ).

Vicarious calibration of TIRS by the TIRS calibration team will rely on a set of well known vicarious calibration sites, such as Lake Tahoe, Salton Sea, and Great Lakes. These test sites are all within the continental US, and thus imagery is collected on a regular basis during regular operation of TIRS.

\section{CONCLUSIONS}

The accurate calibration and characterization of TIRS is critical to the successful inclusion of data from this instrument into the long-term data stream of Landsat. The calibration system and methodology described here are the first steps in providing a well-understood and accurate sensor. The combination of preflight and in-flight calibration plays a key role to ensure that TIRS meets its calibration accuracy requirements.

\section{REFERENCES}

[1] Williams, D.L., S.N. Goward, and T.J. Arvidson. "Landsat: Yesterday, Today, and Tomorrow,” Photogrammetric Engineering \& Remote Sensing, vol. 72, pp: 1171-1178, 2006.

[2] Thome, K. B. Markham, J. Barker, P. Slater, and S. Biggar, "Radiometric calibration of Landsat," Photogramm. Eng. and Remote Sensing., Vol. 63, pp. 853-858, 1997.

[3] Markham, B. L. and J. L. Barker, eds, Special issue: LIDQA Final Symposium, Photogram. Eng. and Rem. Sens., 51:12451493, 1985. 